\title{
Yield Of Bronchoalveolar Lavage In Suspected Pulmonary TB With Negative Induced-Sputum Smear
}

\author{
Javid Ahmad Malik, Ramees Mohi Ud Din Mir, Shabir Ahmad Lone, M. Umar Majid, \\ Majid Khalil Rather, Nisar Hajam, Bilal Ahangar.
}

Department of Pulmonary Medicine, SKIMS Medical College Srinagar, India

\section{A B S T R A C T}

Background: Bronchoalveolar lavage (BAL) has been used to recover mycobacterium tuberculosis (MTB) from the respiratory tract of patients with lung infiltrates as nearly $50 \%$ of pulmonary tuberculosis (PTB) cases are sputum smear negative for acid fast bacilli (AFB). We prospectively compared the diagnostic yield of induced sputum (IS) smear with BAL smear and culture to assess its role in the early diagnosis of sputum negative PTB.

Methods: We performed bronchoscopy to obtain BAL in 52 consecutive patients of suspected PTB between December 2013 and November 2015 after negative results on 2 samples of IS smears. BAL samples were stained with Ziehl-Neelsen (ZN) stain and cultured on L-J media.

Results: Cohort of 52 IS negative patients had a mean age of 46 years (SD 15.6) with $58 \%$ male participants. Comorbidities were present in $31 \%$ participants whereas $42 \%$ were current smokers. Tuberculosis was the final diagnosis in $37 \%(n=19)$ patients and rest $63 \%(n=33)$ had nontubercular etiologies like bronchiectasis $(n=6)$, pneumonia $(n=5)$, interstitial lung disease $(n=3)$, lung abscess $(n=2)$, lung cancer $(n=1)$, PCP $(n=1)$, hydatid cyst $(n=2)$, pneumonia $(n=5)$ and non- specific infection $(n=13)$. In tuberculosis group $33 \%$ patients had bronchoscopic abnormalities. Tuberculosis was confirmed by BALMTB culture in $90 \%(n=17)$ and by biopsy in $10 \%(n=2)$.

Conclusion: BAL has high diagnostic yield and should be considered in the evaluation of suspected PTB. Bronchoscopy also picked up alternative diagnoses. JMS 2016; 19(2):75-81.

Keywords: Fiberoptic bronchoscopy (FOB), Bronchoalveolar lavage (BAL), Tuberculosis, Induced sputum

\section{INTRODUCTION}

Worldwide tuberculosis (TB) particularly pulmonary tuberculosis (PTB) is a public health problem of immense proportions with main burden on developing countries which are resource limited. Detection of acid-fast bacilli (AFB) in respiratory specimens is recommended by World Health Organization (WHO) as the initial approach to the diagnosis of $\mathrm{PTB}^{1}$ but in those patients who cannot produce sputum spontaneously this method has a low sensitivity., Though relatively easy to perform sputum microscopy and culture can be negative in a significant proportion of PTB patients with sensitivities ranging from 25 to $45 \%{ }^{4 \cdot 6}$

\section{Correspondence}

Dr. Javid Ahmad Malik, M.D.

Associate Professor and Head, Department of

Pulmonary Medicine, SKIMS Medical College, Srinagar, India e-mail: javaidmalik2009@yahoo.co.in
Hypertonic saline induced sputum (IS) has been shown to increase the diagnostic yield of sputum examination. In the diagnosis of PTB both in terms of higher microscopy and higher culture positivity, results from various studies have confirmed the higher diagnostic yield of bronchoscopy over sputum examination. ${ }^{10-12}$ In contrast some studies have shown no additional yield of bronchoscopy over IS..$^{13,14}$ Nearly $50 \%$ of PTB cases are sputum smear negative for $\mathrm{AFB}^{15}$ and the transmission rate of smear negative TB has been reported as high as $22 \% .{ }^{16}$ Some studies suggest that close to $50 \%$ of sputum smear negative patients would ultimately need anti- tuberculosis therapy (ATT) if left untreated. ${ }^{17,18}$ Therefore samples other than sputum have an important role in patients with occult tuberculosis or other conditions that mimic TB. Unanswered question is whether an invasive investigation like bronchoscopy is must or should we treat tuberculosis empirically on clinico- 
radiological grounds particularly in tuberculosis endemic countries. Empiric treatment may not only result in unnecessary cost and toxicity of ATT but we may also miss other mimicking diseases particularly malignancy. To address this dilemma we designed the present study to answer whether BAL obtained with bronchoscopy provides any additional diagnostic yield after at least two inducedsputum smear tests were reported negative in patients suspected of having active PTB.

\section{METHODS}

In the current study we prospectively evaluated 52 consecutive cases between December 2013 and November 2015, aged 20 years and above who had respiratory symptoms and radiographic findings suspicious of PTB but were unable to produce sputum spontaneously and had at least two IS reports negative for AFB. Written informed consent was obtained from all eligible participants. Exclusion criteria included previous history of PTB, severe COPD, pregnancy and respiratory or cardiac failure. Detailed history, physical examination, complete blood count, coagulation profile, HIV serology and CT scan chest were done in all the patients included in the study. Patients suspected of PTB were first evaluated with induced-sputum smear specimen for AFB on at least two consecutive days. Morning induced sputum (IS) collection was performed by using 3\% hypertonic saline nebulized through a mouthpiece using an ultrasonic nebulizer for at least 20 minutes. Samples were then centrifuged and concentrated and subjected to AFB smear examination.

Standard bronchoscopic premedication protocol published earlier by us was used ${ }^{19}$ however we minimized the use of lidocaine as much as possible because of its known antimicrobial properties. ${ }^{20}$ We obtained BAL specimens by wedging the bronchoscope into the radiologically most involved pulmonary segment and instilling four to six aliquots of $20 \mathrm{ml}$ normal saline and aspirated until a total of 30 to $40 \mathrm{ml}$ of fluid return was obtained. Using standard techniques all specimens were stained with Ziehl- Neelsen (ZN) stain for AFB and cultured in LöwensteinJensen (LJ) medium for the detection of mycobacterium tuberculosis (MTB). Definite PTB was defined as a positive culture for MTB in bronchoalveolar lavage fluid (BALF). All the participants were followed for at least 6 months after enrollment in the study.

\section{RESULTS}

Cohort of 52 patients included in the present study had a mean age of 46 years (SD 15.6) with 58\% male participants (Tables 1 and 2). Comorbidities mainly diabetes, chronic kidney and liver diseases were present in $31 \%$ of our patients whereas $42 \%$ participants were current smokers. Tuberculosis was the final diagnosis in 19 (37\%) patients and rest 33 (63\%) had pneumonia (CAP), interstitial lung disease (ILD), lung abscess, infected hydatid cyst or nonspecific pulmonary infection (NSPI). There was no difference in the comparative age of the study subjects in relation to final diagnosis (Table 3 ). The main presenting symptoms cough, fever, hemoptysis and weight loss were not significantly different in tubercular and non- tubercular patients $(\mathrm{p}>0.8)$. In tuberculosis group 33\% patients had bronchoscopic abnormalities like endobronchial nodularity, mucosal irregularity and increased secretions in the radiographically involved lung. These patients had tuberculosis finally confirmed either by BALMTB culture $17(90 \%)$ or by bronchial biopsy or transbronchial lung biopsy $(10 \%)$ demonstrating caseating granulomas consistent with tuberculosis (Table 4). The two patients who had only biopsy evidence of PTB responded to six months anti-tuberculosis therapy with complete clinical and radiological improvement. All those patients who had BALAFB positive grew MTB on culture. In three patients a final diagnosis of ILD was made on the basis of TBLB and radiological findings. A 43 year chronic smoker was diagnosed to have small cell lung cancer from bronchial biopsy. This gentleman had fleeting infiltrates in serial radiographs and endobronchial growth in left upper lobe bronchus. Out of six bronchiectasis patients pseudomonas was isolated from BAL fluid of three. Two young males who had cavitation in upper lobes, hydatid membranes intrabronchially and positive hydatid serology had a final diagnosis of infected hydatid cysts. One 55 year old male smoker who had poorly controlled diabetes with diffuse ground glass opacity (GGO) on high resolution CT (HRCT) scan of chest had pneumocystis jirovici previously known as PCP in BAL fluid. Five patients were found to have community acquired pneumonia (CAP) and two lung abscess. We finally labeled 13 (25\%) patients who did not fit into any known clinical entity as cases of nonspecific pulmonary infection (NSPI) because bronchoscopy and BAL fluid analysis could not reveal any specific etiology 
and there was complete clinical improvement on follow up with antibiotic therapy. No BAL specimen with a positive AFB smear or positive mycobacterial culture result was identified as MOTT or NTM. Both IS and BAL procedures were well tolerated by most patients and there were no major complication with either of the two procedures.

TABLE 1: Demographic features of the study populationTuberculosis vs. Non- tuberculosis patients:

\begin{tabular}{|c|c|c|c|c|c|c|}
\hline & & $\begin{array}{c}\text { TB group } \\
(n=19)\end{array}$ & & $\begin{array}{l}\text { n-TB group } \\
(n=33)\end{array}$ & $\begin{array}{c}\text { Total } \\
(n=52)\end{array}$ & $P$ value \\
\hline Age $\geq 50$ years & & (52.6\%) & 14 & $(42.4 \%)$ & $24(46.2 \%)$ & 0.477 \\
\hline Male gender & & (57.9\%) & 19 & $57.6 \%$ & $3057.7 \%$ & 0.982 \\
\hline Smoker & 9 & $47.4 \%$ & 13 & $39.4 \%$ & $22 \quad 42.3 \%$ & 0.575 \\
\hline Co-morbidity & 7 & $36.8 \%$ & 9 & $27.3 \%$ & $1630.8 \%$ & 0.7472 \\
\hline \multicolumn{7}{|l|}{ Presentation: } \\
\hline Cough & 10 & $(52.6 \%)$ & 17 & (51.5\%) & 27 (51.9\%) & 0.938 \\
\hline Fever & 4 & $21.1 \%$ & 8 & $24.2 \%$ & $1223.1 \%$ & 0.793 \\
\hline Hemoptysis & 4 & $21.1 \%$ & 5 & $15.2 \%$ & $9 \quad 17.3 \%$ & 0.588 \\
\hline Weight loss & 1 & $5.3 \%$ & 3 & $9.1 \%$ & $4 \quad 7.7 \%$ & 0.618 \\
\hline
\end{tabular}

TABLE 2: Demographic features of the study populationTuberculosis vs. Non- tuberculosis patients:

\begin{tabular}{|l|c|c|c|c|}
\hline \multicolumn{1}{|c|}{ Age (years) } & N & Mean & SD & Range \\
\hline Tub erculosis patients & 19 & 47.00 & 14.57 & $21-74$ \\
Non-Tuberculosis patients & 33 & 45.42 & 16.32 & $22-74$ \\
Total & 52 & 46.00 & 15.58 & $21-74$ \\
\hline P= 0.729 & & & & \\
\hline
\end{tabular}


TABLE 3: Comparative age of the study subjects in relation to final diagnosis:

\begin{tabular}{|l|c|c|c|c|c|c|c|c|c|}
\hline $\begin{array}{l}\text { Age } \\
\text { (years) }\end{array}$ & $\begin{array}{c}\text { Tuberculosis } \\
\mathrm{N}=19\end{array}$ & $\begin{array}{c}\text { ILD } \\
\mathrm{N}=3\end{array}$ & $\begin{array}{c}\text { Bronchiectasis } \\
\mathrm{N}=6\end{array}$ & $\begin{array}{c}\text { Lung } \\
\text { Cancer } \\
\mathrm{N}=1\end{array}$ & $\begin{array}{c}\text { PCP } \\
\mathrm{N}=\end{array}$ & $\begin{array}{c}\text { CAP } \\
\mathrm{N}=5\end{array}$ & $\begin{array}{c}\text { Hydatid } \\
\text { cyst }\end{array}$ & $\begin{array}{c}\text { Lung } \\
\text { abscess }\end{array}$ & $\begin{array}{c}\mathrm{NSPI} \\
\mathrm{N}=\end{array}$ \\
\hline Median & 51.00 & 56.00 & 27.50 & & & 49.00 & 28.00 & 25.00 & 60.00 \\
\hline Mean & 47.00 & 55.33 & 28.50 & 43 & 55 & 49.80 & 28.00 & 25.00 & 54.54 \\
\hline SD & 14.57 & 4.04 & 6.09 & & & 13.92 & 4.24 & 2.83 & 15.22 \\
\hline Range & $21-74$ & $51-59$ & $22-39$ & & & $31-70$ & $25-31$ & $23-27$ & $28-74$ \\
\hline
\end{tabular}

CAP: community acquired pneumonia

ILD: interstitial lung disease

NSPI: nonspecific pulmonary infection

TABLE 4: Diagnostic methods used in the study populationTuberculosis vs. Non- tuberculosis patients:

\begin{tabular}{l|c|c|c|c|c|c|c|} 
& \multicolumn{2}{|c|}{$\begin{array}{c}\text { TB group } \\
(\mathrm{n}=19)\end{array}$} & \multicolumn{2}{c|}{$\begin{array}{c}\text { Non-TB group } \\
(\mathrm{n}=33)\end{array}$} & \multicolumn{2}{c|}{$\begin{array}{c}\text { Total } \\
(\mathrm{n}=52)\end{array}$} & $\begin{array}{c}\text { P } \\
\text { value }\end{array}$ \\
\hline Abnormalities on FOB & 6 & $31.6 \%$ & 9 & $27.3 \%$ & 15 & $29.4 \%$ & 0.650 \\
BAL-AFB +ve & 9 & $47.4 \%$ & 0 & $0.0 \%$ & 9 & $17.3 \%$ & $<0.001$ \\
₹ BAL-MTB culture +ve & 17 & $89.5 \%$ & 0 & $0.0 \%$ & 17 & $32.7 \%$ & $<0.001$ \\
(c) Biopsy--TBLB / BB & 6 & & 5 & & 11 & & -
\end{tabular}

- $\quad$ BAL: bronchoalveolar lavage, TBLB: transbronchial lung biopsy, BB: bronchial biopsy,

- $\quad$ MTB: mycobacterium tuberculosis

- $\quad ¥$ [out of 19 TB patients 17 (90\%) had MTB culture positive and remaining 2 (10\%) had diagnosis based on biopsy].

- $\quad$ (C) [Biopsy was done only in those patients where it was judged to be important during bronchoscopy. In TB group, 4 patients had both culture and biopsy confirmation of TB whereas 2 had only biopsy confirmation of TB. In non-TB group, 3 biopsies revealed ILD, one small cell lung cancer and one biopsy was normal].

\section{DISCUSSION}

Pulmonary tuberculosis (PTB) is a communicable disease with adverse public health consequences throughout the world. Nearly $50 \%$ of PTB cases are sputum smear negative for $\mathrm{AFB}{ }^{15}$ and the transmission rate of smear negative TB may be as high as $22 \% .^{16}$ The investigation for sputum negative PTB differs between countries, depending upon economy and prevalence of TB. In countries with a high prevalence of TB and a high clinical suspicion, ATT is often initiated without further investigations. Ideally any patient with suspected PTB on clinical and radiological grounds requires further investigations to confirm the diagnosis. Moreover in view of the global challenge of spread and lethality of MDR and XDR- TB, it is important to stop the practice of empiric treatment in sputum negative cases of suspected PTB. The diagnostic yield of induced sputum is 
certainly more than that of spontaneously expectorated sputum however the unanswered question remains whether bronchoscopy provides any additional diagnostic yield over induced sputum. A study that compared the yield between spontaneous sputum, IS and bronchoscopy in patients with suspected PTB concluded that bronchoscopy had a significantly higher diagnostic yield than IS. ${ }^{21}$ This study also reported that these investigations are complimentary to each other as the yield of any one modality was low and varied from $36 \%$ to $63 \%$.Contrary to this two small studies in patients with negative IS did not find any additional yield by performing bronchoscopy. ${ }^{13,22}$ The lower yield of bronchoscopy in these studies possibly may have resulted from the inexperience of the bronchoscopists and also from the use of lidocaine during bronchoscopy. Lidocaine is not only antibacterial and antifungal but it also has antimycobacterial properties and as little as $1 \mathrm{ml}$ of $2 \%$ lidocaine in the tracheobronchial tree can reduce the culture positivity rate for M. tuberculosis by $50 \% .{ }^{23}$ In the present study we minimized the use of lidocaine and all bronchoscopic procedures were performed by one consultant.

In patients with sputum smear negative PTB, bronchoscopic interventions have been reported to enhance the diagnostic yield. ${ }^{2426}$ Both in terms of higher microscopy and higher culture positivity, results from various studies have confirmed the higher diagnostic yield of bronchoscopy over sputum examination. ${ }^{10-12}$ In the present study we report the diagnostic yield of bronchoscopically obtained BAL fluid in 52 patients with suspected PTB and with at least two negative induced sputum (IS) specimens. Our results suggest that BAL is a useful and largely safe tool in the workup of sputum smear negative PTB.

The diagnosis of PTB in our study was confirmed in $37 \%$ of IS negative patients by BAL and biopsy. This included $90 \%$ by BAL-MTB culture and $10 \%$ by bronchial and transbronchial lung biopsies. Across the studies considerably different yields have been reported regarding the yield of bronchoscopy in the diagnosis of PTB ${ }^{10,11,13,14,26-30}$ reflecting differences in study design and the patient population being studied. Some investigators proceeded directly to bronchoscopy without sputum induction and reported a high diagnostic yield between $32.5 \%{ }^{26}$ and $90 \%{ }^{10}$ for bronchoscopy. In sharp contrast to our 33\% BAL- MTB culture positivity a study from Mayo Clinic, USA by Vivek ${ }^{31}$ reported that bronchoscopy is useful in obtaining culture confirmation of PTB in only $10 \%$ of patients with negative results on multiple IS samples. This huge variation may possibly be due to very low prevalence of tuberculosis in that country. In the present study like an earlier report by Mohan ${ }^{32}$ we have proven clearly the diagnostic utility of bronchoscopy in culture confirmation of TB after negative results on induced- sputum samples.

In countries like India where the prevalence of tuberculosis is high, patients having clinical and radiological picture compatible with TB but a negative sputum smear for AFB usually receive ATT at peripheral hospitals. One may argue in favor of this strategy as it is useful in most cases with the advantage of avoiding an invasive bronchoscopic procedure because smear-negative TB is a common problem in clinical practice. However, our study has exposed an important limitation of this strategy by diagnosing non- tubercular conditions particularly lung cancer and ILD for which ATT will not mean toxicity of unnecessary treatment but at the same time patient may be loosing the vital time by delayed diagnosis of actual disease. Therefore bronchoscopy and its related procedures such as BAL, bronchial and transbronchial lung biopsy is a safe pathway not only for the patient but also for the doctor whenever faced with such a diagnostic dilemma.

\section{CONCLUSIONS}

Based on our results we conclude that, given its high diagnostic yield and safety, bronchoscopy and BAL should be considered in the evaluation of suspected induced sputum negative pulmonary tuberculosis. The usual practice of starting empiric anti- tubercular drugs in such patients should be discouraged because all that looks like TB on clinico-radiological grounds is not actually TB and bronchoscopy can pick up alternative diagnoses.

\section{REFERENCES}

1. World Health Organization. Treatment of tuberculosis: guidelines for national programs. Geneva, Switzerland: World Health Organization 1993.

2. Murray PR, Elmore C, Krogstad DJ. The acid-fast stain: a specific and predictive test for mycobacterial disease. Ann Intern Med 1980; 92: 512-513.

3. Strumpf IJ, Tsang AY, Sayre JW. Re- evaluation of sputum staining for the diagnosis of pulmonary tuberculosis. Am Rev Respir Dis 1979; 119:599-602. 
4. Merrick ST, Sepkowitz KA, Walsh J, Damson L, McKinley P, Jacobs JL. Comparison of induced versus expectorated sputum for diagnosis of pulmonary tuberculosis by acid-fast smear. American Journal of Infection Control 1997; 25: 463-466.

5. Nelson SM, Deike MA, Cartwright CP. Value of examining multiple sputum specimens in the diagnosis of pulmonary tuberculosis. Journal of Clinical Microbiology 1998; 36:467-469.

6. Kim TC, Blackman RS, Heatwole KM, Kim T, Rochester DF. Acid-fast bacilli in sputum smears of patients with pulmonary tuberculosis. Prevalence and significance of negative smears pretreatment and positive smears post-treatment. The American Review of Respiratory Disease 1984; 129: 264-268.

7. Al Zahrani K, Al Jahdali H, Poirier L, Rene P, Menzies D. Yield of smear, culture and amplification tests from repeated sputum induction for the diagnosis of pulmonary tuberculosis. The International Journal of Tuberculosis and Lung Disease 2001; 5: 855-860.

8. Bell D, Leckie V, McKendrick M. Use of the induced sputum procedure in the investigation of smearnegative pulmonary tuberculosis. Clinical Infectious Diseases 2004; 38: 1504-1505

9. Conde MB, Loivos AC, Rezende VM, Soares SL, Mello FC, Reingold AL, et al. Yield of sputum induction in the diagnosis of pleural tuberculosis. American Journal of Respiratory and Critical Care Medicine 2003; 167: 723725.

10. Chawla R, Pant K, Jaggi OP, Chandrashekhar S, Thukral SS. Fibreoptic bronchoscopy in smearnegative pulmonary tuberculosis. European Respiratory Journal 1988; 1: 804-806.

11. Conde MB, Soares SL, Mello FC, Rezende VM, Almeida LL, Reingold AL, et al. Comparison of sputum induction with fiberoptic bronchoscopy in the diagnosis of tuberculosis: experience at an acquired immune deficiency syndrome reference center in Rio de Janeiro, Brazil. American Journal of Respiratory and Critical Care Medicine 2000; 162: 2238-2240.

12. Ganguly KC, Hiron MM, Mridha ZU, Biswas M, Hassan MK, Saha SC, et al. Comparison of sputum induction with broncho-alveolar lavage in the diagnosis of smear-negative pulmonary tuberculosis. Mymensingh Medical Journal 2008; 17: 115-123.
13. McWilliams T, Wells AU, Harrison AC, Lindstrom S, Cameron RJ, Foskin E. Induced sputum and bronchoscopy in the diagnosis of pulmonary tuberculosis. Thorax 2002; 57: 10101014.

14. Anderson C, Inhaber N, Menzies D. Comparison of sputum induction with fiber-optic bronchoscopy in the diagnosis of tuberculosis. American Journal of Respiratory and Critical Care Medicine 1995; 152: 15701574.

15. Kvale PA, Johnson MC, Wroblewski DA. Diagnosis of tuberculosis Routine cultures of brochial washings are not indicated. Chest 1979;76:140-2.

16. Dener SJ, Bower VS. Diagnosis of pulmonary tuberculosis by flexible bronchoscopy. Am Rev Respir Dis 1979;119:677-9.

17. Hong Kong Chest Service / Tuberculosis Research Center Madras / British Medical Research Council. Sputum smear negative tuberculosis: Controlled clinical trial of 3 month and 2 month regimen of chemotherapy (first report). Lancet 1979; 1:1361-3.

18. Hong Kong Chest Service / Tuberculosis Research Center Madras/ British Medical Research Council. A Study of the characteristics and course of sputum smear negative pulmonary tuberculosis. Tubercle $1981 ; 62: 155-67$.

19. Javid Ahmad Malik, Dheeraj Gupta, Ashutosh N. Agarwal, Surinder K. Jindal. Anticholinergic Premedication for Flexible Bronchoscopy A Randomized, Double-Blind, Placebo-Controlled Study of Atropine and Glycopyrrolate CHEST 2009; 136:347-354.

20. Chandan SS, Faoagali J, Wainwright CE. Sensitivity of respiratory bacteria to lignocaine. Pathology 2005; 37 : 305-307.

21. Schoch OD, Rieder P, Tueller C, Altpeter E, Zellweger JP, Rieder HL, et al. Diagnostic yield of sputum, induced sputum, and bronchoscopy after radiologic tuberculosis screening. American Journal of Respiratory and Critical Care Medicine 2007; 175: 8086.

22. Brown M, Varia H, Bassett P, Davidson RN, Wall R, Pasvol G. Prospective study of sputum induction, gastric washing, and bronchoalveolar lavage for the diagnosis of pulmonary tuberculosis in patients who are unable to expectorate. Clinical Infectious Diseases 
2007; 44: 1415-1420.

23. Conte, B.A. and Laforet, E.G. The role of the topical anesthetic agent in modifying bacteriologic data obtained by bronchoscopy. The New England Journal of Medicine 1962; 267: 957-960.

24. Araz O, Akgun M, Saglam L, Ozden, K, Mirici A. The diagnostic value of bronchoscopy in smear negative cases with pulmonary tuberculosis. Tuberkuloz ve toraks 2008; 56: 150-157.

25. Cary JF. Usefulness of bronchoscopy in the diagnosis of tuberculosis. Chest 1992; 101:292-293.

26. Charoenratanakul S, Dejsomritrutai W, Chaiprasert A. Diagnostic role of fiberoptic bronchoscopy in suspected smear negative pulmonary tuberculosis. Respiratory Medicine 1995; 89: 621-623.

27. Willcox PA, Potgieter PD, Bateman ED, Benatar SR. Rapid diagnosis of sputum negative miliary tuberculosis using the flexible fibreoptic bronchoscope. Thorax 1986; 41: 681-684.

28. Wong CF, Yew WW, Chan CY, Au LY, Cheung SW, Cheng AF. Rapid diagnosis of smear-negative pulmonary tuberculosis via fibreoptic bronchoscopy: utility of polymerase chain reaction in bronchial aspirates as an adjunct to transbronchial biopsies. Respiratory Medicine 1998;92: 815-819.

29. Kennedy DJ, Lewis WP, Barnes PF. Yield of bronchoscopy for the diagnosis of tuberculosis in patients with human immune -deficiency virus infection. Chest 1992; 102: 1040-1044.

30. Jett JR, Cortese DA, Dines DE. The value of bronchoscopy in the diagnosis of mycobacterial disease. A five-year experience. Chest 1981; 80: 575578.

31. Iyer VN, Joshi AY, Boyce TG, et al. Bronchoscopy in suspected pulmonary TB with negative inducedsputum smear and MTD ${ }^{\circledR}$ Gen-probe testing. Respiratory Medicine 2011; 105(7): 1084-1090.

32. Mohan A, Sharma SK. Fibreoptic bronchoscopy in the diagnosis of sputum smear-negative pulmonary tuberculosis: current status. The Indian Journal of Chest Diseases \& Allied Sciences 2008; 50: 67-78. 